\section{Bravo für diese Unterstuitzung in der Fortbildung!}

Ziel der obligatorischen Fortbildung ist das Vertiefen, Erweitern und Verbessern der beruflichen Kenntnisse, Fähigkeiten und Fertigkeiten. Dabei soll medizinischen und technischen Fortschritten sowie den Bedürfnissen der Bevölkerung Rechnung getragen werden. Die Ärztinnen und Ärzte bestimmen Bedarf und Inhalt ihrer Fortbildung selbst. Der «Hippokratest» ermöglicht im deutschsprachigen Raum zum ersten Mal sowohl eine Selbstbeurteilung als auch eine vergleichende Einschätzung der individuellen Fortbildungsbedürfnisse. Der Test erfüllt dadurch eine von der Kommission für Weiter- und Fortbildung seit mehreren Jahren aufgestellte Forderung.
Den Autoren möchte ich zum guten Produkt gratulieren und wünsche diesem eine rasche und breite Akzeptanz. Dadurch werden die Veranstalter von Fortbildungen gefordert, Lernziele zu formulieren und die entsprechenden Inhalte zu vermitteln. Der Test selbst kann weiterentwickelt werden und zur weiteren Unterstützung von Ärztinnen und Ärzte beim Lernen in Ergänzung zu den Fortbildungsveranstaltungen auf breitanerkannte Literatur verweisen.

Max Giger,

Präsident der Kommission für Weiter- und Fortbildung der FMH

\title{
Selbstassessment für Grundversorger und Wegweiser im Fortbildungsdschungel
}

\section{Lucas M. Bachmann}

Résumé en français voir page suivante

Korrespondenz:

PD Dr. med. Lucas M. Bachmann MKM Medizinwissen AG

Poststrasse 15

CH-6300 Zug

Tel. 0417104747

Fax 0417109581

info@mkm-medizinwissen.com

www.mkm-medizinwissen.com

www.hippokratest.com

\section{Zusammenfassung}

Seit September 2008 steht allen niedergelassenen Grundversorgern eine neue Dienstleistung zur Verfügung: der vollständig unabhängig von Pharmafirmen, Medizinprodukteherstellern, Krankenkassen und anderen medizinischen Dienstleistern entwickelte «Hippokratest». Zu einem Preis von 295 Franken erlaubt «Hippokratest» zum einen die Überprüfung des Wissens in den 15 für die Grundversorger relevanten Fachgebieten. Andererseits wird - basierend auf dem offiziellen Fortbildungsprogramm - ein Fortbildungsvorschlag unterbreitet, der allfällige Wissenslücken optimal abdeckt. Die FMH hat das Programm als «FMH-approved» zertifiziert und vergibt nach Durchführung des Assessments 3 Kreditpunkte. Die Schweizerische Gesellschaft für Allgemeinmedizin SGAM unterstützt dieses Programm, dem es das Q-Label verliehen hat, ebenfalls: Absolventen können die gesamte Fortbildungsdauer als Credits anrechnen. Nebst dem Assessment steht eine umfassende Fortbildungsdatenbank mit Veranstaltungen aus der Schweiz, Österreich und Deutschland zur Verfügung, die kostenlos eingesehen werden kann.

\section{Hintergrund}

Der Artikel 3 der geltenden Fortbildungsordnung empfiehlt, dass ein systematischer Aufbau der Fortbildung anhand verschiedener Schritte durchgeführt werden soll. Insbesondere soll ein Defizit an Wissen und Können erkannt werden, entsprechend Fortbildungen ausgewählt werden und eine Selbstkontrolle des Lernerfolgs durchgeführt werden. Leider entspricht die heutige Fortbildungsrealität dieser sinnvollen Vorgabe nicht: Es ist dem Arzt vollkommen selbst überlassen, in welcher Form er seine Fortbildung absolviert. Auf ihrer Website schreibt die Schweizerische Gesellschaft für Innere Medizin SGIM: «Für die Auswahl der Fortbildungsangebote im Rahmen der nachzuweisenden Fortbildung ist jeder Internist selbst

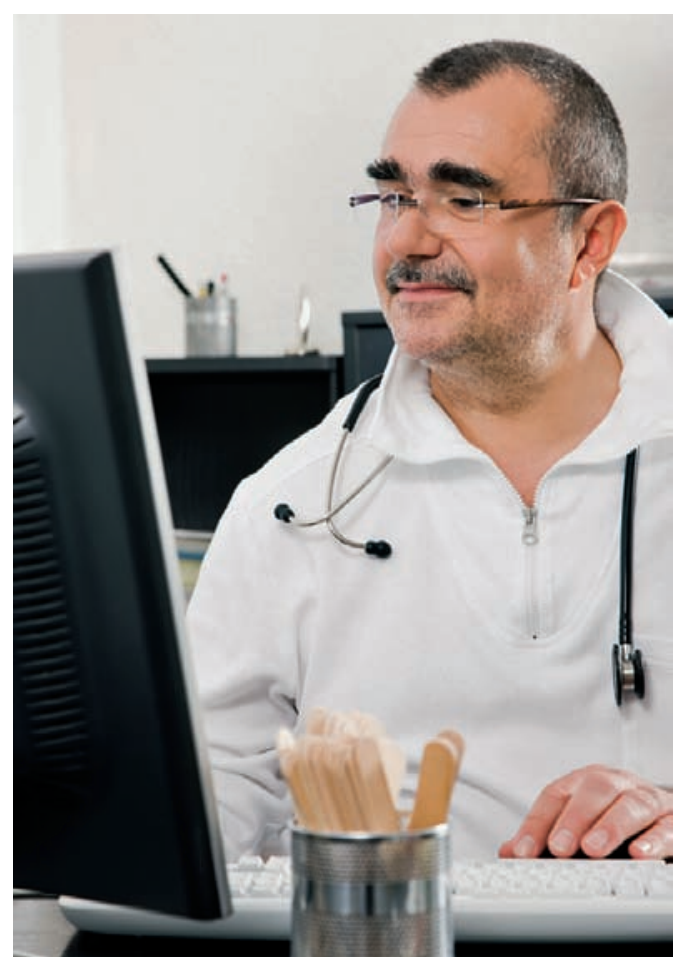

«Hippokratest»: online den eigenen Wissenslücken auf der Spur. 
verantwortlich. Oberstes Ziel soll sein, Wissens-, Fertigkeits- und Fähigkeitslücken zu schliessen und die neu erworbenen Kenntnisse im täglichen beruflichen Umfeld umzusetzen.»

Weiter fordert die SGIM: «Bei der Auswahl der Fortbildungsangebote ist darauf zu achten, dass häufig auftretende Krankheiten, gesundheitliche Probleme und Fragestellungen im Zusammenhang mit der Inneren Medizin entsprechend abgedeckt werden. Auch Grenzgebiete, respektive klinische Nachbargebiete der Inneren Medizin, deren Kenntnisse zur Ausübung einer umfassenden internistischen Tätigkeit im ambulanten (Grundversorgung) und stationären Bereich notwendig sind, müssen bei der Auswahl der Fortbildung berücksichtigt werden.»

Leider wird eine systematische, unabhängige und hochqualifizierte Wissensevaluation im Bereich der Medizin heute nicht angeboten. In der Praxis erfolgt deshalb die Auswahl von Fortbildungsveranstaltungen bei vielen Ärzten nach dem Opportunitätsprinzip. Viele Ärzte besuchen Fortbildungen zur Selbstbestätigung ihres Wissens und weniger zur Behebung ihrer Wissenslücken. Zudem wird die Wahl einer Fortbildung häufig unter Berücksichtigung des Rahmenprogramms und der Liste der Teilnehmer und weniger nach inhaltlichen Aspekten getroffen.

Das ist auch aus anderem Grunde nachvollziehbar: Das Fortbildungsangebot nur schon in der Schweiz ist für den Allgemeinpraktiker oder Internisten riesig gross und unstrukturiert: Neben den regionalen Fortbildungsangeboten werden allein in den drei deutschschweizerischen Universitätskliniken jährlich schätzungsweise 3000 Fortbildungsveranstaltungen, von denen mindestens die Hälfte für Internisten und Hausärzte potentiell relevant ist, angeboten.

Die rasche medizinische Entwicklung und die hohe Dichte an Innovationen machen aber eine effiziente Fortbildungskultur unabdingbar. Während noch vor hundert Jahren die diagnostischen und therapeutischen Möglichkeiten des Arztes sehr beschränkt waren, sieht sich der Arzt von heute bedingt durch die Entwicklungen von bildgebenden Verfahren, Labortests, Genanalysen und medikamentösen Behandlungen einem komplexen Versorgungssystem gegenübergestellt. Die Tendenz, auch komplexe therapeutische Verfahren heute vermehrt in der niedergelassenen Praxis zu verschreiben und schwerer Erkrankte häufiger ambulant zu versorgen, steigert den Fortbildungsbedarf zusätzlich. Das heisst: Die Anforderungen an das Wissen des Allgemeinpraktikers und Internisten steigen, aber es fehlt eine unabhängige, fachlich qualifizierte Möglichkeit, nicht vorhandenes Wissen schnell, verläss-

\section{L'Hippocratest fait office} d'auto-évaluation

\section{pour les médecins généralistes}

Depuis le mois de septembre 2008, tous les médecins de premier recours exerçant en pratique privée ont la possibilité d'effectuer l'Hippocratest. Ce test a été élaboré en toute indépendance, sans ingérence des firmes pharmaceutiques, fabricants de produits thérapeutiques, caisses-maladie et autres fournisseurs de prestations. Pour la somme de 295 francs, tout médecin généraliste peut procéder à une vérification de ses connaissances dans 15 disciplines importantes pour la médecine de premier recours. Parallèlement à cela, et sur la base du programme de formation continue officiel, le médecin concerné reçoit une proposition de formation continue lui indiquant comment combler au mieux ses éventuelles lacunes. La FMH a approuvé ce programme en lui apposant son logo et octroie 3 crédits de formation continue à quiconque se soumet à la procédure. La Société suisse de médecine générale (SSMG) soutient ce programme auquel elle a attribué son label de qualité. Les candidats à cette formation continue peuvent la faire valoir dans son intégralité. En plus du test d'auto-évaluation, les médecins peuvent également accéder gratuitement à une banque de données où figurent toutes les manifestations de formation continue de Suisse, d'Autriche et d'Allemagne.

lich und ohne geschäftliche Hintergründe festzustellen.

\section{Wissensassessment und Fortbildungs- vorschlag: Hippokratest}

Seit September 2008 steht für niedergelassene Grundversorger und andere Interessierte zu einem Preis von 295 Franken nun ein webbasiertes SelfAssessment-Instrument zur Evaluation des individuellen fachspezifischen Wissens und der Lernbedürfnisse zur Verfügung: der Hippokratest. Die Inhalte von «Hippokratest» sind vollständig unabhängig von Interessen der Pharmafirmen, Medizinproduktehersteller, Krankenkassen und anderen medizinischen Dienstleister. Das Instrument wurde von ausgewiesenen Schweizer Fachleuten 
aus 15 verschiedenen Disziplinen (Kardiologie, Psychosomatik, Pneumologie u.a.) konzipiert. Alle Fragen wurden von einem unabhängigen internationalen Expertenbeirat auf ihre Eignung hin überprüft. Hippokratest umfasst 60 MultipleChoice-Fragen mit Einfach- und Mehrfachantwort und erhebt zudem, wie sicher man sich bei der Beantwortung der Fragen gefühlt hat (Confidence Scores). Im Anschluss an das Assessment erhält man unverzüglich eine Fragenauswertung und die anonymisierten Vergleichswerte der Kollegen, die ebenfalls den Test absolviert haben.

Wer hinter dem Hippokratest steht: MKM Medizinwissen AG

Die MKM Medizinwissen AG (MKM steht für Medical Knowledge Management) mit Sitz in Zug wurde von den sechs Gesellschaftern Dr. med. Lucas M. Bachmann, Dr. Jürg R. Conzett, Dr. Urs Läubli, Dr. Andreas Ritter, Andreas M. Simmen und Prof. Dr. med. Johann Steurer Ende 2007 gegründet. Die Firma setzt sich für zukunftsweisende Entwicklungen im Bereich der medizinischen Wissensüberprüfung, -vermittlung und -beratung ein. MKM Medizinwissen entwickelt für Ärzte und medizinische Organisationen massgeschneiderte Strategien zur Verbesserung des Informations- und Wissensmanagements. Oberste Priorität der MKM Medizinwissen AG ist - neben höchster Qualität der angebotenen Dienstleistungen - die vollständige inhaltliche und finanzielle Unabhängigkeit von Pharmafirmen, Medizinprodukteherstellern sowie Krankenkassen und anderen medizinischen Dienstleistern.
Aufgrund des Stärken- und Schwächenprofils wird vom System - basierend auf dem aktuellen nationalen Fortbildungskalender - ein massgeschneiderter Fortbildungsvorschlag gemacht.

Die FMH hat das Programm als «FMH-approved» zertifiziert und vergibt nach Durchführung des Assessments 3 Kreditpunkte. Die Schweizerische Gesellschaft für Allgemeinmedizin SGAM unterstützt dieses Programm, dem es das Q-Label verliehen hat, ebenfalls: Absolventen können die gesamte Fortbildungsdauer als Credits anrechnen.

Das Assessment ist vollständig anonym, d.h. niemand ausser dem Nutzer/der Nutzerin kann die persönlichen Leistungsdaten einsehen. Die Daten werden mit modernen Verschlüsselungsverfahren an einen Datenserver gesandt, um dort - anonymisiert - geschützt gespeichert zu werden. Dies erlaubt den Vergleich der einzelnen Leistungsdaten mit den aggregierten Daten der Fachkollegen. Mehrjährige Nutzer haben zudem die Möglichkeit, ihre Leistungsdaten im zeitlichen Verlauf darzustellen, um Wissensverbesserungen zu analysieren.

\section{Aktualität und Qualität}

Der Hippokratest wird jedes Jahr aktualisiert. Jeweils ab Januar des aktuellen Jahres wird ein neuer Fragenkatalog aufgeschaltet. Dies steigert die Attraktivität für diejenigen, welche den Test jedes Jahr durchführen wollen. Es werden nur Fragen verwendet, welche von ausgewiesenen Fachleuten erstellt wurden und von einem Gremium von Allgemeinmedizinern als relevant eingestuft wurden. Dieses zweistufige Verfahren verhindert, dass für die Allgemeinmedizin irrelevante Fragen gestellt werden. Beide Gremien arbeiten unabhängig und erhalten für ihre Tätigkeit eine finanzielle Unterstützung. 